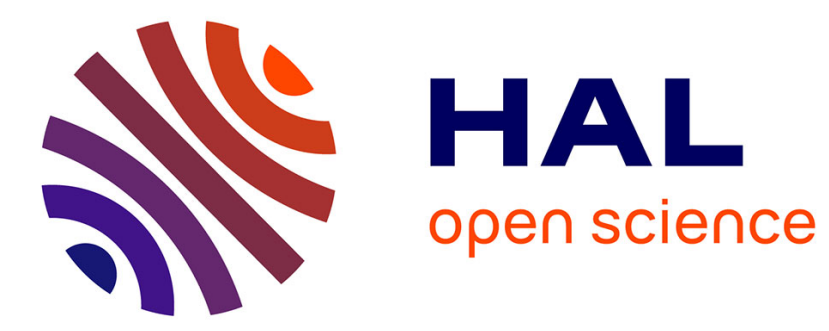

\title{
On Stochastic Bounds for Monotonic Processor Sharing Networks
}

Thomas Bonald, Alexandre Proutière

\section{To cite this version:}

Thomas Bonald, Alexandre Proutière. On Stochastic Bounds for Monotonic Processor Sharing Networks. Queueing Systems, 2004, 10.1023/B:QUES.0000032802.41986.c6 . hal-01285972

\section{HAL Id: hal-01285972 \\ https://hal.science/hal-01285972}

Submitted on 10 Mar 2016

HAL is a multi-disciplinary open access archive for the deposit and dissemination of scientific research documents, whether they are published or not. The documents may come from teaching and research institutions in France or abroad, or from public or private research centers.
L'archive ouverte pluridisciplinaire HAL, est destinée au dépôt et à la diffusion de documents scientifiques de niveau recherche, publiés ou non, émanant des établissements d'enseignement et de recherche français ou étrangers, des laboratoires publics ou privés. 


\title{
On stochastic bounds for monotonic processor sharing networks
}

\author{
T. Bonald and A. Proutière \\ France Telecom R\&D \\ $\{$ Thomas. Bonald, Alexandre.Proutiere\}@francetelecom.com
}

June 3,2003

\begin{abstract}
We consider a network of processor sharing nodes with independent Poisson arrival processes. Nodes are coupled through their service capacity in that the speed of each node depends on the number of customers present at this and any other node. We assume the network is monotonic in the sense that removing a customer from any node increases the service rate of any customer. Under this assumption, we give stochastic bounds on the number of customers present at any node. We also identify limiting regimes that allow to test the tightness of these bounds. The bounds and the limiting regimes are insensitive to the service time distribution. We apply these results to a number of practically interesting systems, including the discriminatory processor sharing queue, the generalized processor sharing queue, and data networks whose resources are shared according to max-min fairness. Keywords: Processor sharing networks, stochastic bounds, balance, monotonicity, insensitivity.
\end{abstract}

\section{Introduction}

The processor sharing (PS) service discipline has traditionally been used to represent the way jobs share the processing unit of a computer system [17]. More recently, it has been used to represent the bandwidth sharing of a bottleneck link in the Internet [21]. It is well known that, assuming Poisson arrivals, the stationary distribution of the number of customers in a PS node depends on the distribution of service requirements through the mean only. This insensitivity property is practically interesting as robust engineering rules can be developed for the corresponding systems independently of precise statistics on the requests (job sizes or data transfer volumes). The property extends to networks of PS nodes where the service capacity of each node depends on the number of customers present at this node $[1,14]$. The steady-state distribution then has a well-known product-form expression. More generally, the steady state distribution of a network of PS nodes whose service capacity depends on the whole network state has a closed-form expression and is insensitive to the distribution of service requirements provided the following balance property is satisfied in any state [23]:

"For all pairs of nodes $i, j$, the relative change in the service capacity of node $i$ when a customer present at node $j$ is removed is the same as the relative change in the service capacity of node $j$ when a customer present at node $i$ is removed."

Applying these results to the context of data networks, we have identified the way flows should ideally share link capacities so that flow level performance depends on traffic statistics through the traffic intensity on each network route only [7]. The corresponding allocation, referred to as "balanced fairness", differs from well-known "max-min fairness" where flow rates are made as equal as possible $[2,20]$. 
Such queueing models are strictly applicable only if resources are shared in a perfectly fair way. This is naturally not the case of real systems. Each job in a computer system is typically split into a sequence of elementary tasks that are scheduled in a more or less fair way by the processing unit. Similarly, each flow in a data network consists of a sequence of packets whose sending rate is adapted in response to congestion indications such as packet losses, typically under the control of TCP. Realized bandwidth sharing then depends on many factors including the packet scheduling and buffer management schemes implemented in the routers and is typically unfair to those TCP flows having long round-trip times. Thus the performance of real systems is never exactly that of the above ideal queueing networks. In particular, it is somehow sensitive to precise statistics on service requests. The objective of the present paper is to provide mathematical tools for evaluating the sensitivity of real systems and studying the extent to which the results derived from ideal queueing networks provide a sufficiently accurate approximation of their performance to be used as practical engineering guidelines.

Specifically, we consider networks of PS nodes whose service capacity depends on the whole network state but that do not necessarily satisfy the above balance property. The only required property is the following:

"Removing a customer from any node increases the service rate of all customers."

Note that this monotonicity property is satisfied by many real systems. In particular, the model is sufficiently general to account for unfair service disciplines such as discriminatory processor sharing (DPS) or generalized processor sharing (GPS) where the service capacity is shared according to some predetermined weights. It can also be used to represent max-min fair bandwidth sharing of a data network having a tree topology for instance, as shown below. We derive stochastic bounds on the performance of monotonic PS networks by means of sample-path comparisons with balanced networks. We also identify the limiting regimes obtained when nodes operate at very different time-scales in order to quantify the sensitivity of the network and to assess the tightness of these bounds. The bounds and the limiting regimes are shown to be insensitive to the distribution of service requirements.

Little is known about the performance of non-balanced PS networks. This may simply be explained by the fact that the balance property is actually a necessary and sufficient condition for insensitivity [6]. Thus the performance of non-balanced networks cannot be evaluated without specific assumptions on service requirements. Usual Markovian techniques apply of course, assuming i.i.d. exponential or Cox service requirements, but generally only yield numerical results and then only for systems with a relatively small state space. Large deviations techniques, which have notably been applied to the study of the GPS queue $[3,8,19]$, are appropriate to quantify the occurence of rare events [11] but useless for evaluating simple performance metrics such as mean response times. Analysis in heavy traffic provides interesting asymptotic results $[18,25]$ but has proved successful for specific networks only, despite some recent advances in the area [16]. A more promising approach, which has notably been applied to the analysis of 2-class PS queues with priority $[9,22]$, consists in identifying the above mentioned limiting regimes obtained when nodes/classes operate at very different time-scales, e.g., by means of singular perturbation techniques [24]. The question of whether these limiting regimes constitute bounds or not remains largely open, however.

In the next section, we describe the considered PS networks and introduce the notions of balance and monotonicity. The main results are given in the following two sections, where we derive stochastic bounds for monotonic PS networks and identify the limiting regimes. These results are illustrated in Section 5 on a number of examples, including the DPS and GPS queues. Section 6 concludes the paper. 


\section{Processor sharing networks}

We consider an open network of $N$ processor sharing (PS) nodes. Customers arrive at each node $i$ as a Poisson process of intensity $\lambda_{i}$, require i.i.d. services of mean $\sigma_{i}$, and leave the network once served. We denote by $\rho_{i}=\lambda_{i} \sigma_{i}$ the traffic intensity at node $i$ and $x_{i}$ the number of customers present at node $i$. Nodes are coupled through their service capacity in that the speed of each node $i$ is a function $\phi_{i}(x)$ of the state $x=\left(x_{1}, \ldots, x_{N}\right)$. We assume that $\phi_{i}(x)>0$ if and only if $x_{i}>0$.

Notation. We denote by $e_{i}$ the unit vector with 1 in component $i$ and 0 elsewhere, for $i=1, \ldots, N$. For $x, y \in \mathbb{R}^{N}$, we use the notation $x \leq y$ if $x_{i} \leq y_{i}$ for $i=1, \ldots, N$ and $|x|=\sum_{i=1}^{N} x_{i}$. We denote by $\leq_{s t}$ the strong stochastic ordering on $\mathbb{R}^{N}$-valued random variables, i.e., $X \leq{ }_{s t} Y$ if and only if $E[f(X)] \leq E[f(Y)]$ for all increasing functions $f$.

\subsection{Balance property}

We first recall the main properties of so-called "balanced" processor sharing networks. For a full account readers are referred to [23].

Balance property. We say that the network is balanced if for all pairs of nodes $i, j$ :

$$
\frac{\phi_{i}\left(x-e_{j}\right)}{\phi_{i}(x)}=\frac{\phi_{j}\left(x-e_{i}\right)}{\phi_{j}(x)}, \quad \forall x: x_{i}>0, x_{j}>0 .
$$

Consider the function $\Phi$ recursively defined by $\Phi(0)=1$ and:

$$
\Phi(x)=\frac{\Phi\left(x-e_{i}\right)}{\phi_{i}(x)}, \quad x_{i}>0 .
$$

The balance property implies that this defines a unique function $\Phi$, referred to as the balance function. Note that $\Phi(x)$ may be viewed as the weight of any direct path from state 0 to state $x$, where a direct path is a set of consecutive states $x(0) \equiv 0, x(1), x(2), \ldots, x(n) \equiv x$ such that $x(k)-x(k-1)=e_{i(k)}$ for some $i(k), k=1, \ldots, n$, with $n=|x|$, and the weight of such a path is the inverse of the product of $\phi_{i(k)}(x(k))$ for $k=1, \ldots, n$ (refer to Figure 1 ).

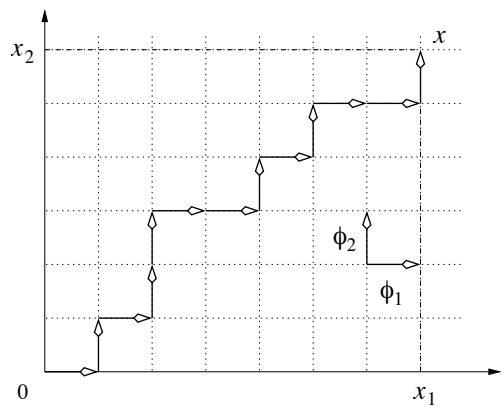

Figure 1: The balance function $\Phi(x)$ is equal to the weight of any direct path from state 0 to state $x$.

The steady state distribution is then given by:

$$
\pi(x)=\pi(0) \times \Phi(x) \prod_{i=1}^{N} \rho_{i}^{x_{i}},
$$


where $\pi(0)$ is given by the usual normalizing condition, provided:

$$
\sum_{x} \Phi(x) \prod_{i=1}^{N} \rho_{i}^{x_{i}}<\infty .
$$

Note that $\pi$ depends on the distribution of service requirements at any node $i$ through the mean $\sigma_{i}$ only. The steady state distribution is also insensitive to the time-scale of each node in that it depends on the arrival rate $\lambda_{i}$ and the mean service requirement $\sigma_{i}$ through their product $\rho_{i}$ only. It turns out that a PS network for which one of these insensitivity properties holds is necessarily balanced [6].

\subsection{Monotonicity}

We are interested in evaluating the performance of non-balanced networks, for which the stationary distribution is sensitive to service requirements and typically unknown. The method consists in deriving stochastic bounds for "monotonic" networks by means of sample-path comparisons.

Monotonicity. We say that the network is monotonic if for all nodes $i$ :

$$
\frac{\phi_{i}(x)}{x_{i}} \geq \frac{\phi_{i}(y)}{y_{i}}, \quad \forall x \leq y: x_{i}>0
$$

Denote by $X(t)$ the vector of the number of customers at each node at time $t \geq 0$ given the network is empty at time 0 . Note that the stochastic process $\{X(t), \Sigma(t)\}_{t \geq 0}$ where $\Sigma(t)=\left(\Sigma_{1}(t), \ldots, \Sigma_{N}(t)\right)$ and $\Sigma_{i}(t)$ is a $X_{i}(t)$-dimensional vector representing the remaining service requirement of customers at node $i$ at time $t$, is an irreducible Markov process.

Lemma 1 Assume that the network with service capacities $\phi_{i}, i=1, \ldots, N$, is monotonic. Denote by $\tilde{X}(t)$ the vector of the number of customers at each node at time $t \geq 0$ starting empty at time 0 for service capacities $\tilde{\phi}_{i}, i=1, \ldots, N$, satisfying $\tilde{\phi}_{i}(x) \leq \phi_{i}(x)$ for any $i$ in any state $x$ such that $x_{i}>0$. Then $X(t) \leq \tilde{X}(t)$ for all $t \geq 0$.

Proof. The proof follows from the fact that for any states $x \leq \tilde{x}$ and any node $i$ such that $x_{i}>0$ :

$$
\frac{\phi_{i}(x)}{x_{i}} \geq \frac{\phi_{i}(\tilde{x})}{\tilde{x}_{i}} \geq \frac{\tilde{\phi}_{i}(\tilde{x})}{\tilde{x}_{i}}
$$

\section{Insensitive stochastic bounds}

In this section, we consider a monotonic PS network and derive insensitive lower and upper bounds by means of sample-path comparisons with balanced networks. The bounds coincide if the network is itself balanced.

\subsection{Lower bound}

Denote by $\check{X}(t)$ the vector of the number of customers at each node at time $t \geq 0$ starting empty at time 0 , for those service capacities $\check{\phi}_{1}, \ldots, \check{\phi}_{N}$ corresponding to the balance function $\check{\Phi}$ recursively defined by $\check{\Phi}(0)=1$ and:

$$
\check{\Phi}(x)=\max _{i: x_{i}>0} \frac{\check{\Phi}\left(x-e_{i}\right)}{\phi_{i}(x)} .
$$


Note that $\check{\Phi}(x)$ is the weight of the direct path of maximum weight from state 0 to state $x$ (cf. $\S 2.1$ ). This provides a lower bound on performance (expressed in terms of response time for instance) in view of the following result.

Theorem 1 If the network is monotonic, then $X(t) \leq \check{X}(t)$ for all $t \geq 0$.

Proof. The proof follows from Lemma 1 and the fact that for any node $i$ in any state $x$ such that $x_{i}>0$ :

$$
\check{\phi}_{i}(x)=\frac{\check{\Phi}\left(x-e_{i}\right)}{\check{\Phi}(x)} \leq \phi_{i}(x) \text {. }
$$

By definition, the network with service capacities $\check{\phi}_{1}, \ldots, \check{\phi}_{N}$ is balanced. In particular, its steady state distribution is insensitive and given by:

$$
\check{\pi}(x)=\check{\pi}(0) \times \check{\Phi}(x) \prod_{i=1}^{N} \rho_{i}^{x_{i}}
$$

provided:

$$
\sum_{x} \check{\Phi}(x) \prod_{i=1}^{N} \rho_{i}^{x_{i}}<\infty .
$$

In view of Theorem 1, the ergodicity of the Markov process $\{\check{X}(t), \check{\Sigma}(t)\}_{t \geq 0}$ implies that of $\{X(t), \Sigma(t)\}_{t \geq 0}$. Denoting by $X$ and $\check{X}$ the corresponding stationary vectors of the number of customers at each node, we get $X \leq_{s t} \check{X}$.

\subsection{Upper bound}

Denote by $\hat{X}(t)$ the vector of the number of customers at each node at time $t \geq 0$ starting empty at time 0 , for the service capacities $\hat{\phi}_{1}, \ldots, \hat{\phi}_{N}$ corresponding to the balance functions $\hat{\Phi}$ recursively defined by $\hat{\Phi}(0)=1$ and

$$
\hat{\Phi}(x)=\min _{i: x_{i}>0} \frac{\hat{\Phi}\left(x-e_{i}\right)}{\phi_{i}(x)} .
$$

Note that $\hat{\Phi}(x)$ is the weight of the direct path of minimum weight from state 0 to state $x$ (cf. $\S 2.1$ ). This provides an upper bound on performance in view of the following result.

Theorem 2 If the network is monotonic, then $X(t) \geq \hat{X}(t)$ for all $t \geq 0$.

Proof. As for Theorem 1, the proof follows from Lemma 1 and the fact that for any node $i$ in any state $x$ such that $x_{i}>0$ :

$$
\hat{\phi}_{i}(x)=\frac{\hat{\Phi}\left(x-e_{i}\right)}{\hat{\Phi}(x)} \geq \phi_{i}(x) .
$$

Again, the network with service capacities $\hat{\phi}_{1}, \ldots, \hat{\phi}_{N}$ is balanced. In particular, its steady state distribution is insensitive and given by:

$$
\hat{\pi}(x)=\hat{\pi}(0) \times \hat{\Phi}(x) \prod_{i=1}^{N} \rho_{i}^{x_{i}}, \quad \text { provided } \quad \sum_{x} \hat{\Phi}(x) \prod_{i=1}^{N} \rho_{i}^{x_{i}}<\infty .
$$


In view of Theorem 2, the ergodicity of the Markov process $\{X(t), \Sigma(t)\}_{t \geq 0}$ implies that of $\{\hat{X}(t), \hat{\Sigma}(t)\}_{t \geq 0}$. Denoting by $X$ and $\hat{X}$ the corresponding stationary vectors of the number of customers at each node, we get $X \geq_{s t} \hat{X}$.

\subsection{Bias property}

The lower and upper bounds derived above are entirely determined by the balance functions $\check{\Phi}$ and $\hat{\Phi}$. It remains difficult in general to find an explicit expression for these functions. A notable exception is the case of "biased" networks.

Bias property. We say that the network is biased if nodes can be numbered in such a way that for all pairs of nodes $i, j, i \leq j$ :

$$
\frac{\phi_{i}\left(x-e_{j}\right)}{\phi_{i}(x)} \geq \frac{\phi_{j}\left(x-e_{i}\right)}{\phi_{j}(x)}, \quad \forall x: x_{i}>0, x_{j}>0
$$

For biased networks, we simply have:

$$
\check{\Phi}(x)=\left(\prod_{n=1}^{x_{N}} \phi_{N}\left(n e_{N}\right) \times \prod_{n=1}^{x_{N-1}} \phi_{N-1}\left(n e_{N-1}+x_{N} e_{N}\right) \times \ldots \times \prod_{n=1}^{x_{1}} \phi_{1}\left(n e_{1}+x_{2} e_{2}+\ldots+x_{N} e_{N}\right)\right)^{-1}
$$

and

$$
\hat{\Phi}(x)=\left(\prod_{n=1}^{x_{1}} \phi_{1}\left(n e_{1}\right) \times \prod_{n=1}^{x_{2}} \phi_{2}\left(x_{1} e_{1}+n e_{2}\right) \times \ldots \times \prod_{n=1}^{x_{N}} \phi_{N}\left(x_{1} e_{1}+\ldots+x_{N-1} e_{N-1}+n e_{N}\right)\right)^{-1} .
$$

Note that $\check{\Phi}(x)$ and $\hat{\Phi}(x)$ correspond to the weight of straight paths (for $N=2$, from state 0 to state $x$ through state $\left(0, x_{2}\right)$ and state $\left(x_{1}, 0\right)$, respectively, cf. Figure 1$)$.

\subsection{Local behavior}

We now identify the local behavior of the bounds when the traffic intensity at all nodes except one tends to zero. These results will prove useful in assessing the tightness of the bounds (see Theorem 4 below). Assume that the stability condition (6) holds. Let $Y_{i}$ be the random variable describing the steady-state number of customers in node $i$ in isolation, i.e., when the traffic intensity at any other node is equal to zero. Denote by $\zeta_{i}$ the distribution of $Y_{i}$, given by:

$$
\zeta_{i}(y)=\zeta_{i}(0) \prod_{k=1}^{y} \frac{\rho_{i}}{\phi_{i}\left(k e_{i}\right)}, \quad y \in \mathbb{N} .
$$

Proposition 1 For any positive function $f$ such that $E[f(\check{X})]<\infty$, we have:

$$
\lim _{\rho_{j} \rightarrow 0, j \neq i} E[f(\check{X})]=\lim _{\rho_{j} \rightarrow 0, j \neq i} E[f(\hat{X})]=E\left[f\left(Y_{i} e_{i}\right)\right]
$$

Proof. In view of (5) and (7), we have:

$$
E[f(\check{X})]=\frac{\sum_{x} f(x) \check{\Phi}(x) \prod_{i=1}^{N} \rho_{i}^{x_{i}}}{\sum_{x} \check{\Phi}(x) \prod_{i=1}^{N} \rho_{i}^{x_{i}}} \quad \text { and } \quad E[f(\hat{X})]=\frac{\sum_{x} f(x) \hat{\Phi}(x) \prod_{i=1}^{N} \rho_{i}^{x_{i}}}{\sum_{x} \hat{\Phi}(x) \prod_{i=1}^{N} \rho_{i}^{x_{i}}}
$$


The proof then follows from the fact that in each of these two expressions, each term of the numerator and the denominator is an increasing function of $\rho_{1}, \ldots, \rho_{N}$ and

$$
\check{\Phi}\left(y e_{i}\right)=\hat{\Phi}\left(y e_{i}\right)=\frac{1}{\prod_{k=1}^{y} \phi_{i}\left(k e_{i}\right)}, \quad y \geq 0 .
$$

We now derive first-order terms of $E[f(\check{X})]$ and $E[f(\hat{X})]$ in $\rho_{j}, j \neq i$, when the traffic intensity at any node except node $i$ tends to zero, provided the following "local bias" property holds.

Local bias property. We say that there is a local bias of node $i$ against node $j$ if:

$$
\frac{\phi_{i}\left(x-e_{j}\right)}{\phi_{i}(x)} \leq \frac{\phi_{j}\left(x-e_{i}\right)}{\phi_{j}(x)}, \quad \forall x: x=x_{i} e_{i}+e_{j}, x_{i}>0
$$

Similarly, we say that there is a local bias of node $i$ in favor of node $j$ if:

$$
\frac{\phi_{i}\left(x-e_{j}\right)}{\phi_{i}(x)} \geq \frac{\phi_{j}\left(x-e_{i}\right)}{\phi_{j}(x)}, \quad \forall x: x=x_{i} e_{i}+e_{j}, x_{i}>0
$$

Note that for biased networks, there is a local bias of any node $i$ against any node $j \leq i$ and in favor of any node $j \geq i$.

Proposition 2 Consider a positive function $f$ such that $E[f(\check{X})]<\infty$ and $f(x)=0$ for all states $x$ such that $x_{j}=0$. If there is a local bias of some node $i$ against node $j$, then:

$$
\lim _{\rho_{j^{\prime}} \rightarrow 0, j^{\prime} \neq i} \frac{E[f(\check{X})]}{\rho_{j}}=\sum_{y=0}^{\infty} \frac{f\left(y e_{i}+e_{j}\right)}{\phi_{j}\left(y e_{i}+e_{j}\right)} \zeta_{i}(y) .
$$

If there is a local bias of some node $i$ in favor of node $j$, then:

$$
\lim _{\rho_{j^{\prime}} \rightarrow 0, j^{\prime} \neq i} \frac{E[f(\hat{X})]}{\rho_{j}}=\sum_{y=0}^{\infty} \frac{f\left(y e_{i}+e_{j}\right)}{\phi_{j}\left(y e_{i}+e_{j}\right)} \zeta_{i}(y) .
$$

Proof. In view of (5) and (7), we have:

$$
\frac{E[f(\check{X})]}{\rho_{j}}=\frac{\sum_{x: x_{j}>0} f(x) \check{\Phi}(x) \rho_{j}^{x_{j}-1} \prod_{j^{\prime} \neq j} \rho_{j^{\prime}}^{x_{j^{\prime}}}}{\sum_{x} \check{\Phi}(x) \prod_{j^{\prime}} \rho_{j^{\prime}}^{x_{j^{\prime}}}}
$$

and

$$
\frac{E[f(\hat{X})]}{\rho_{j}}=\frac{\sum_{x: x_{j}>0} f(x) \hat{\Phi}(x) \rho_{j}^{x_{j}-1} \prod_{j^{\prime} \neq j} \rho_{j^{\prime}}^{x_{j^{\prime}}}}{\sum_{x} \hat{\Phi}(x) \prod_{j^{\prime}} \rho_{j^{\prime}}^{x_{j^{\prime}}}}
$$

The proof then follows from the fact that in each of these two expressions, each term of the numerator and the denominator is an increasing function of $\rho_{1}, \ldots, \rho_{N}$, while

$$
\check{\Phi}\left(y e_{i}\right)=\hat{\Phi}\left(y e_{i}\right)=\frac{1}{\prod_{k=1}^{y} \phi_{i}\left(k e_{i}\right)}, \quad y \geq 0,
$$


and

$$
\check{\Phi}\left(y e_{i}+e_{j}\right)=\frac{1}{\phi_{j}\left(y e_{i}+e_{j}\right) \prod_{k=1}^{y} \phi_{i}\left(k e_{i}\right)}, \quad y \geq 0,
$$

if there is a local bias of node $i$ against node $j$,

$$
\hat{\Phi}\left(y e_{i}+e_{j}\right)=\frac{1}{\phi_{j}\left(y e_{i}+e_{j}\right) \prod_{k=1}^{y} \phi_{i}\left(k e_{i}\right)}, \quad y \geq 0
$$

if there is a local bias of node $i$ in favor of node $j$.

\subsection{Equivalent capacity}

The notion of "equivalent capacity" is convenient for evaluating network performance in terms of mean response times. We define the equivalent capacity $\gamma_{i}$ of node $i$ as the ratio of the mean service requirement $\sigma_{i}$ to the mean response time $E\left[T_{i}\right]$. Applying Little formula, we get:

$$
\gamma_{i}=\frac{\rho_{i}}{E\left[x_{i}\right]} .
$$

Denote by $\check{\gamma}_{i}$ and $\hat{\gamma}_{i}$ the equivalent capacity of node $i$ for the lower and upper bounds derived in $\S 3.1$ and $\S 3.2$, respectively. For any monotonic system such that the stability condition (6) holds, it follows from Theorems 1 and 2 that:

$$
\check{\gamma}_{i} \leq \gamma_{i} \leq \hat{\gamma}_{i}
$$

Denoting by $\gamma_{i}(s)$ the ratio of $s$ to the mean response time $E\left[T_{i}(s)\right]$ of a customer requiring a service $s>0$ at node $i$, we have in fact the stronger result:

$$
\check{\gamma}_{i} \leq \gamma_{i}(s) \leq \hat{\gamma}_{i}, \quad \forall s>0 .
$$

This is due to the property that the mean response time of a customer is proportional to its service requirement in any balanced network [6].

\section{Insensitive limiting regimes}

In this section, we consider any PS network such that the Markov process $\{X(t), \Sigma(t)\}_{t \geq 0}$ is ergodic for some fixed traffic intensities $\rho_{1}, \ldots, \rho_{N}$, independently of $\sigma_{1}, \ldots, \sigma_{N}$. Note that if the network is monotonic, it is sufficient that the stability condition (6) holds in view of Theorem 1 . If the network is not balanced, the stationary distribution $\pi$ of $X(t)$ depends on $\sigma_{1}, \ldots, \sigma_{N}[7$, Corollary 1]. We shall denote explicitly $\pi \equiv \pi_{\sigma_{1}, \ldots, \sigma_{N}}$. We consider the limiting regimes obtained when the mean service requirements at all nodes except one, say node $i$, successively tend to zero. Node $i$ is then referred to as "quasi-stationary" due to the slow variations of the number of customers present at this node compared to any other node. These limiting regimes prove useful in quantifying the sensitivity of the system and the tightness of the bounds derived in previous section. A brief reminder on the convergence of probability measures is given Appendix A. We refer the reader to [4] for more detail.

\subsection{Limiting distribution}

Without loss of generality, we let $\sigma_{1}, \sigma_{2}, \ldots, \sigma_{N-1}$ successively tend to 0 with fixed traffic intensities $\rho_{1}, \rho_{2}, \ldots, \rho_{N-1}$. The following result is proved in Appendix B 
Theorem 3 Assume that the family of probability measures $\pi_{\sigma_{1}, \ldots, \sigma_{N}}$ is tight. Then $\pi_{\sigma_{1}, \ldots, \sigma_{N}} \Rightarrow \bar{\pi}$ when $\sigma_{1}, \sigma_{2}, \ldots, \sigma_{N-1}$ successively tend to 0 with fixed traffic intensities $\rho_{1}, \rho_{2}, \ldots, \rho_{N-1}$, where $\bar{\pi}$ is the probability measure given by:

$$
\bar{\pi}(x)=\prod_{i=1}^{N} \bar{\pi}_{i}\left(x_{i}, \ldots, x_{N}\right) .
$$

For all $i=1, \ldots, N, \bar{\pi}_{i}\left(\cdot, x_{i+1}, \ldots, x_{N}\right)$ is the probability measure defined by:

$$
\bar{\pi}_{i}\left(x_{i}, x_{i+1}, \ldots, x_{N}\right)=\bar{\pi}_{i}\left(0, x_{i+1}, \ldots, x_{N}\right) \times \prod_{y=1}^{x_{i}} \frac{\rho_{i}}{\bar{\phi}_{i}\left(y, x_{i+1}, \ldots, x_{N}\right)},
$$

with $\bar{\phi}_{1} \equiv \phi_{1}$ and for $i=2, \ldots, N$,

$$
\bar{\phi}_{i}\left(x_{i}, \ldots, x_{N}\right)=\sum_{x_{1}, \ldots, x_{i-1}} \phi_{i}(x) \prod_{j=1}^{i-1} \bar{\pi}_{j}\left(x_{j}, \ldots, x_{N}\right) .
$$

It is worth noting that the limiting regime is insensitive in the sense that $\bar{\pi}$ is independent of the distribution of service requirements. The following result is a direct consequence of Theorem 3 .

Corollary 1 Assume that the family of probability measures $\pi_{\sigma_{1}, \ldots, \sigma_{N}}$ is tight. Denote by $X_{\sigma_{1}, \ldots, \sigma_{N}} a$ random variable with distribution $\pi_{\sigma_{1}, \ldots, \sigma_{N}}, \bar{X}$ a random variable with distribution $\bar{\pi}$. Let $f$ be any increasing function. If $E[f(\bar{X})]=\infty$, then

$$
\lim _{\sigma_{N-1} \rightarrow 0} \ldots \lim _{\sigma_{2} \rightarrow 0} \lim _{\sigma_{1} \rightarrow 0} E\left[f\left(X_{\sigma_{1}, \ldots, \sigma_{N}}\right)\right]=\infty .
$$

If $X_{\sigma_{1}, \ldots, \sigma_{N}}$ is $f$-uniformly integrable, then

$$
\lim _{\sigma_{N-1} \rightarrow 0} \ldots \lim _{\sigma_{2} \rightarrow 0} \lim _{\sigma_{1} \rightarrow 0} E\left[f\left(X_{\sigma_{1}, \ldots, \sigma_{N}}\right)\right]=E[f(\bar{X})] .
$$

Proposition 3 If the network is monotonic and inequality (6) holds, the family of probability measures $\pi_{\sigma_{1}, \ldots, \sigma_{N}}$ is tight. In addition, for any increasing function $f$ such that $E[f(\check{X})]<\infty, X_{\sigma_{1}, \ldots, \sigma_{N}}$ is $f$ uniformly integrable.

Proof. For any $\varepsilon>0$, there exists $n$ such that:

$$
\operatorname{Pr}(|\check{X}|>n) \leq \varepsilon \quad \text { and } \quad E\left[f(\check{X}) \mathbb{I}_{\{|\check{X}|>n\}}\right] \leq \varepsilon .
$$

The proof then follows from the fact that $X_{\sigma_{1}, \ldots, \sigma_{N}} \leq_{s t} \check{X}$.

\subsection{Local behavior}

Let $\bar{\pi}$ be the limiting distribution obtained when some node $i$ is quasi-stationary. We identify the local behavior obtained when the traffic intensity at any other node tends to zero. The following result, proved in Appendix B, is independent of the way $\bar{\pi}$ is obtained, i.e., of the order in which the mean service requirements at nodes $j \neq i$ successively tend to zero. We denote by $\bar{X}$ a random variable with distribution $\bar{\pi}$. 
Theorem 4 Consider a monotonic network. For any increasing function $f$ such that $E[f(\bar{X})]<\infty$,

$$
\lim _{\rho_{j} \rightarrow 0, j \neq i} E[f(\bar{X})]=E\left[f\left(Y_{i} e_{i}\right)\right] .
$$

If $E[f(\check{X})]<\infty$ and for some $j \neq i, f(x)=0$ for all states $x$ such that $x_{j}=0$,

$$
\lim _{\rho_{j^{\prime}} \rightarrow 0, j^{\prime} \neq i} \frac{E[f(\bar{X})]}{\rho_{j}}=\sum_{y=0}^{\infty} \frac{f\left(y e_{i}+e_{j}\right)}{\phi_{j}\left(y e_{i}+e_{j}\right)} \zeta_{i}(y) .
$$

\subsection{Equivalent capacity}

Let $\check{\gamma}_{j}^{i}$ be the equivalent capacity of node $j$ when node $i$ is quasi-stationary. It follows from Theorem 4 and Proposition 1 that:

$$
\lim _{\rho_{j} \rightarrow 0, j \neq i}\left(\bar{\gamma}_{i}^{i}-\check{\gamma}_{i}\right)=0 \quad \text { and } \quad \lim _{\rho_{j} \rightarrow 0, j \neq i}\left(\bar{\gamma}_{i}^{i}-\hat{\gamma}_{i}\right)=0 .
$$

If there is a local bias of node $i$ against node $j$, then in view of Proposition 2,

$$
\lim _{\rho_{j^{\prime}} \rightarrow 0, j^{\prime} \neq i}\left(\bar{\gamma}_{j}^{i}-\check{\gamma}_{j}\right)=0
$$

while if there is a local bias of node $i$ in favor of node $j$,

$$
\lim _{\rho_{j^{\prime}} \rightarrow 0, j^{\prime} \neq i}\left(\bar{\gamma}_{j}^{i}-\hat{\gamma}_{j}\right)=0
$$

Thus the lower bound becomes tight when most load is generated by "favored" nodes while the upper bound becomes tight when most load is generated by "penalized" nodes. In particular, we obtain for any biased network:

$$
\lim _{\rho_{j^{\prime}} \rightarrow 0, j^{\prime} \neq N}\left(\bar{\gamma}_{j}^{N}-\check{\gamma}_{j}\right)=0 \quad \text { and } \quad \lim _{\rho_{j^{\prime}} \rightarrow 0, j^{\prime} \neq 1}\left(\bar{\gamma}_{j}^{1}-\hat{\gamma}_{j}\right)=0, \quad \forall j=1, \ldots, N .
$$

\section{$5 \quad$ Examples}

We now apply the results to a number of multiclass systems. Each class will be represented as a node of a PS network. We assume customers of each class arrive as a Poisson process and have i.i.d. service requirements. We denote by $\rho_{i}$ the traffic intensity of class $i$, and by $\rho=\sum_{i=1}^{N} \rho_{i}$ the overall system load. Performance is evaluated in terms of the equivalent capacity of each class. Though the results hold for an arbitrary number of classes, the graphs illustrate simple cases with $N=2$ classes only (except in $\S 5.4$ where a case with $N=3$ classes is also shown). We then have two limiting regimes, one referred to as "Class 1 QS" where class 1 is quasi-stationary, the other one referred to as "Class 2 QS" where class 2 is quasi-stationary. On each graph, simulation results are also plotted for illustration purposes. These results are obtained for i.i.d. exponential services of unit mean for each class.

\subsection{Discriminatory processor sharing queue}

The discriminatory processor sharing (DPS) service discipline consists in sharing the service capacity in proportion to some predetermined weights. Let $w_{i}$ be the weight of class- $i$ customers, $i=1, \ldots, N$, with the convention $w_{1} \leq w_{2} \leq \ldots \leq w_{N}$. A DPS queue corresponds to a PS network with:

$$
\phi_{i}(x)=\frac{w_{i} x_{i}}{w_{1} x_{1}+\ldots+w_{N} x_{N}} .
$$


One easily verifies that this network is monotonic and biased. For $N=2$ classes, we obtain:

$$
\check{\Phi}(x)=\prod_{n=1}^{x_{2}} \frac{w_{1} x_{1}+w_{2} n}{w_{2} n} \quad \text { and } \quad \hat{\Phi}(x)=\prod_{n=1}^{x_{1}} \frac{w_{1} n+w_{2} x_{2}}{w_{1} n}
$$

It turns out that the stability condition (6) does not necessarily hold whenever $\rho<1$, the stability condition of the DPS queue.

For this particular system, explicit expressions have been derived for the mean response time of each class under the assumption of i.i.d.exponential services [12]. As the limiting regimes are insensitive, we deduce from these expressions that the equivalent capacities when class 1 and class 2 are quasi-stationary are respectively given by:

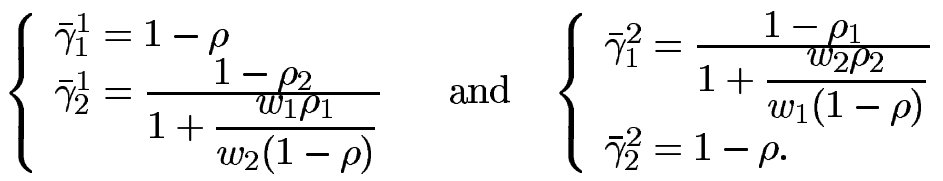

Figure 2 shows the results obtained when $w_{1} / w_{2}=2$ for different load distributions between class 1 and class 2. As expected in view of (9), the lower bound is tighter as more load is generated by class 2 , while the upper bound is tighter as more load is generated by class 1 .

Most load generated by class $1\left(\rho_{1} / \rho=0.9, \rho_{2} / \rho=0.1\right)$
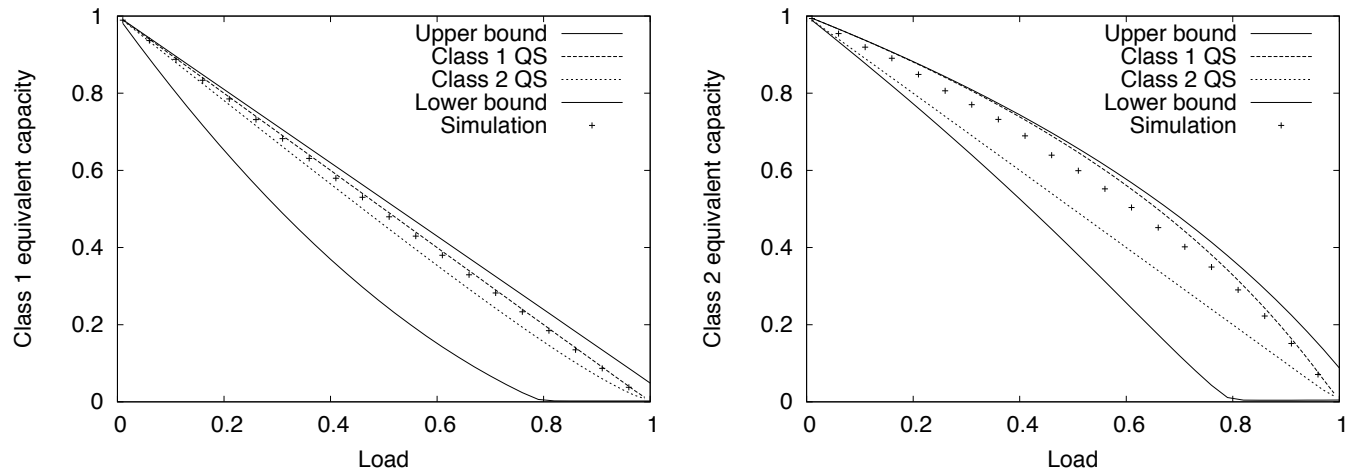

Most load generated by class $2\left(\rho_{1} / \rho=0.1, \rho_{2} / \rho=0.9\right)$
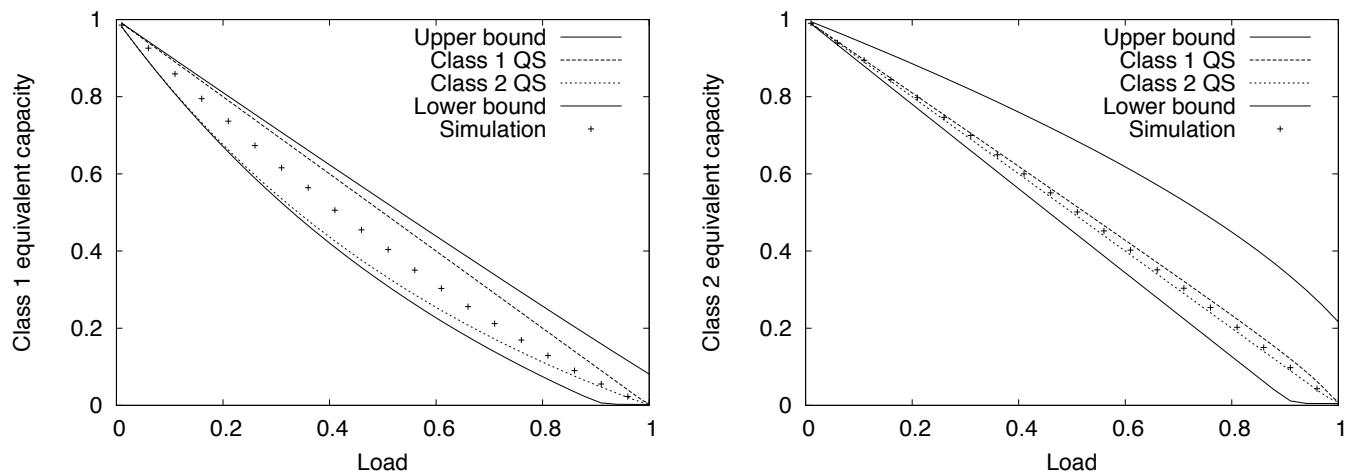

Figure 2: Performance of a 2-class DPS queue. $\left(w_{1} / w_{2}=2\right.$, different load distributions between class 1 and class 2$)$ 


\subsection{Generalized processor sharing queue}

The generalized processor sharing (GPS) service discipline consists in sharing the service capacity between classes in proportion to some predetermined weights. Let $w_{i}$ be the weight of class $i, i=1, \ldots, N$, with the convention $w_{1} \leq w_{2} \leq \ldots \leq w_{N}$ and $w_{1}+w_{2}+\ldots+w_{N}=1$. The system corresponds to a PS network with:

$$
\phi_{i}(x)=\frac{w_{i}}{\sum_{j: x_{j}>0} w_{j}}
$$

This network is monotonic but not biased. There is a local bias of any node $i$ against any node $j \leq i$. We get in any state $x$ such that $x_{i}>0$ for all $i$ :

$$
\check{\Phi}(x)=\frac{w_{1}\left(w_{1}+w_{2}\right) \ldots\left(w_{1}+\ldots+w_{N-1}\right)}{w_{1}^{x_{1}} \ldots w_{N}^{x_{N}}} .
$$

In particular, the stability condition (6) holds if and only if $\rho_{i}<w_{i}$ for $i=1, \ldots, N$. Note that this condition is more restrictive than $\rho<1$, the stability condition of the GPS queue. For $N=2$ classes, we obtain the following normalizing constant, from which the equivalent capacity of each class can easily be deduced:

$$
\sum_{x_{1}, x_{2}} \check{\Phi}\left(x_{1}, x_{2}\right) \rho_{1}^{x_{1}} \rho_{2}^{x_{2}}=1+\frac{\rho_{1}}{1-\rho_{1}}+\frac{\rho_{2}}{1-\rho_{2}}+w_{1} \frac{\rho_{1} \rho_{2}}{\left(w_{1}-\rho_{1}\right)\left(w_{2}-\rho_{2}\right)} .
$$

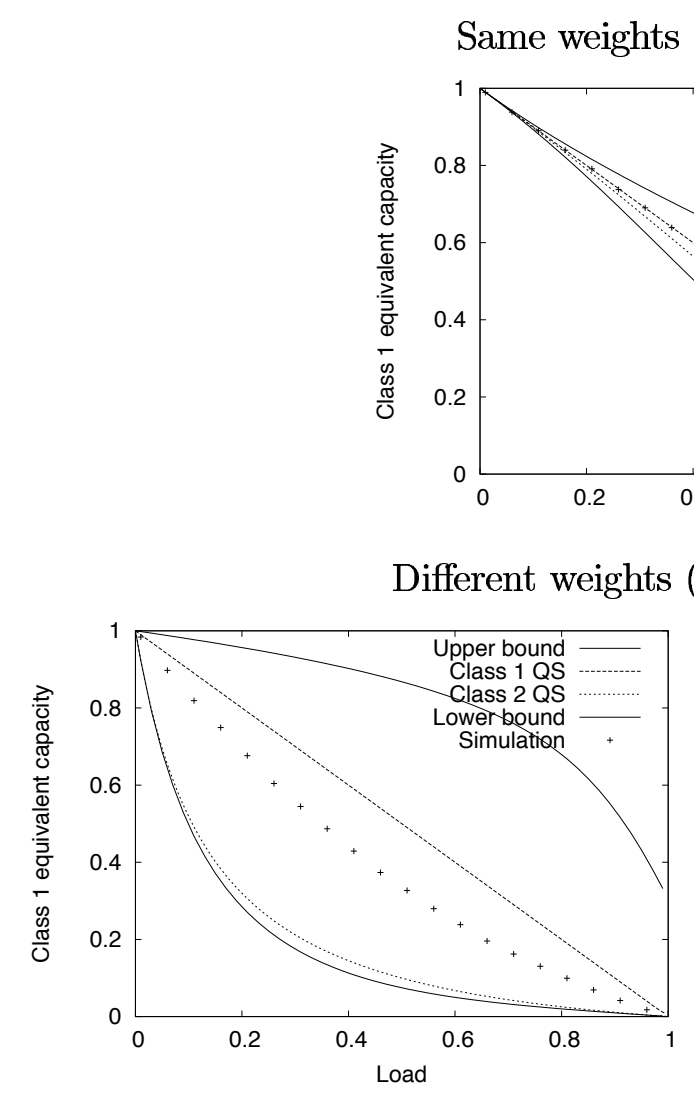

$$
\left.w_{1}=w_{2}=0.5\right)
$$

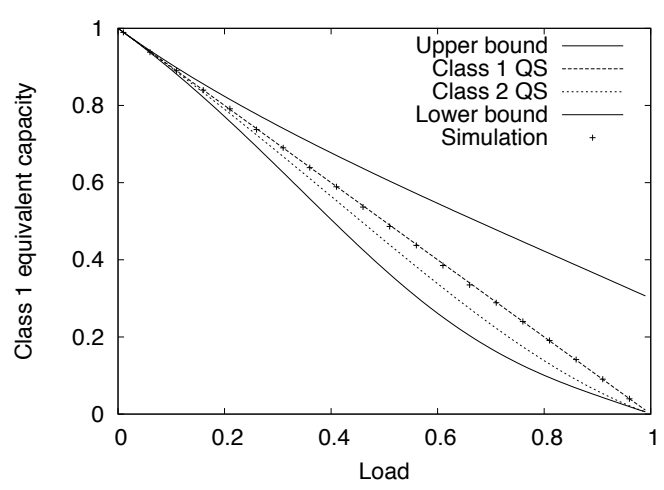

Different weights $\left(w_{1}=0.1, w_{2}=0.9\right)$

Figure 3: Performance of a 2-class GPS queue.

(traffic intensity proportional to the weight: $\rho_{1} / w_{1}=\rho_{2} / w_{2}$ ) 
Concerning the limiting regimes, we get:

$$
\left\{\begin{array} { l } 
{ \overline { \gamma } _ { 1 } ^ { 1 } = 1 - \rho } \\
{ \overline { \gamma } _ { 2 } ^ { 1 } = \frac { 1 - \rho _ { 2 } } { 1 + \frac { w _ { 1 } \rho _ { 1 } } { ( 1 - \rho _ { 2 } ) ( w _ { 2 } - \rho _ { 2 } ) } } }
\end{array} \quad \text { and } \quad \left\{\begin{array}{l}
\bar{\gamma}_{1}^{2}=\frac{1-\rho_{1}}{1+\frac{w_{2} \rho_{2}}{\left(1-\rho_{1}\right)\left(w_{1}-\rho_{1}\right)}} \\
\bar{\gamma}_{2}^{2}=1-\rho .
\end{array}\right.\right.
$$

Figure 3 shows the results obtained when the load distribution is proportional to the weights. As expected in view of $\S 4.3$, the lower bound is tight for class 1 when most load is generated by class 2 . We observe that the system is approximately insensitive for equal weights and highly sensitive when the weights differ significantly.

\subsection{Multirate system}

We now consider a multirate system representing a unit capacity link shared by data flows with rate constraints, i.e., the rate of each flow cannot exceed a fixed value referred to as its access rate. The performance of such a system is evaluated in $[6,7]$ for the balanced fair allocation. Here we consider the max-min fair allocation $[2,20]$. Max-min fairness can be obtained using the following "filling" procedure:

Starting from zero, increase linearly the rate of each flow. When the capacity constraint of one or several flows is reached, freeze the rate of these flows and proceed with this linear filling for those flows not yet constained. Continue the procedure until all flows are constrained.

Let $a_{i}$ be the access rate of class- $i$ flows, with the convention $a_{1} \geq a_{2} \geq \ldots \geq a_{N}$ and $a_{1} \leq 1$. Such a multirate system corresponds to a PS network with:

$$
\phi_{i}(x)=a_{i} x_{i}, \quad \forall i>k \quad \text { and } \quad \phi_{i}(x)=\left(1-\sum_{j>k} a_{j} x_{j}\right) \frac{x_{i}}{\sum_{j \leq k} x_{j}}, \quad \forall i \leq k,
$$

where $k$ is the minimum non-negative integer such that:

$$
a_{k} \sum_{j \leq k} x_{j}+\sum_{j>k} a_{j} x_{j} \leq 1
$$

One easily verifies that this PS network is monotonic and biased. The stability condition (6) holds whenever $\rho<1$ in view of Proposition 4 below and the fact that the service capacity of each class corresponds to that of a PS queue except for a finite number of states:

$$
\phi_{i}(x)=\frac{x_{i}}{\sum_{j} x_{j}} \quad \forall x: a_{N}|x| \geq 1
$$

Proposition 4 Consider two PS networks such that the service capacities coincide except for a finite number of states. If the stability condition (6) holds for one of these two networks, it holds for the other.

Proof. Let $\phi_{1}, \ldots, \phi_{N}$ and $\phi_{1}^{\prime}, \ldots, \phi_{N}^{\prime}$ be service capacities such that $\phi_{i}(x)=\phi_{i}^{\prime}(x)$ for all nodes $i$ in any state $x$ such that $|x|>n$, for some integer $n$. Denote by $\check{\Phi}$ and $\check{\Phi}^{\prime}$ the respective balance functions given by (4). Assume that the stability condition (6) holds for $\check{\Phi}^{\prime}$. Define $\tilde{\Phi}$ as:

$$
\tilde{\Phi}(x)=\alpha \times \check{\Phi}^{\prime}(x) \quad \text { for all } x:|x| \leq n, \quad \tilde{\Phi}(x)=\max _{i: x_{i}>0} \frac{\tilde{\Phi}\left(x-e_{i}\right)}{\phi_{i}(x)} \quad \text { otherwise. }
$$


where

$$
\alpha=\frac{\max _{x:|x| \leq n} \check{\Phi}(x)}{\min _{x:|x| \leq n} \check{\Phi}^{\prime}(x)}
$$

The proof then follows from the fact that $\breve{\Phi}(x) \leq \tilde{\Phi}(x)=\alpha \times \check{\Phi}^{\prime}(x)$ for any state $x$.

For $N=2$ classes with $a_{1}=1$, we obtain:

$$
\check{\Phi}(x)=\prod_{n=1}^{x_{2}} \frac{1}{\min \left(1, n a_{2}\right)} \prod_{n=1}^{x_{1}} \frac{1}{\max \left(\frac{n}{n+x_{2}}, 1-x_{2} a_{2}\right)} \quad \text { and } \quad \hat{\Phi}(x)=\prod_{n=1}^{x_{2}} \frac{1}{\min \left(\frac{n}{x_{1}+n}, n a_{2}\right)} .
$$

Figure 4 shows the results obtained with $a_{1}=1$ and $a_{2}=0.1$. Again, the accuracy of the bounds depends on the load distribution between class 1 and class 2 according to (9). We observe that, as for the DPS queue for which the bias property also holds, the performance of all classes is better when class 1 is quasi-stationary than when class 2 is quasi-stationary.

Most load generated by class $1\left(\rho_{1} / \rho=0.9, \rho_{2} / \rho=0.1\right)$
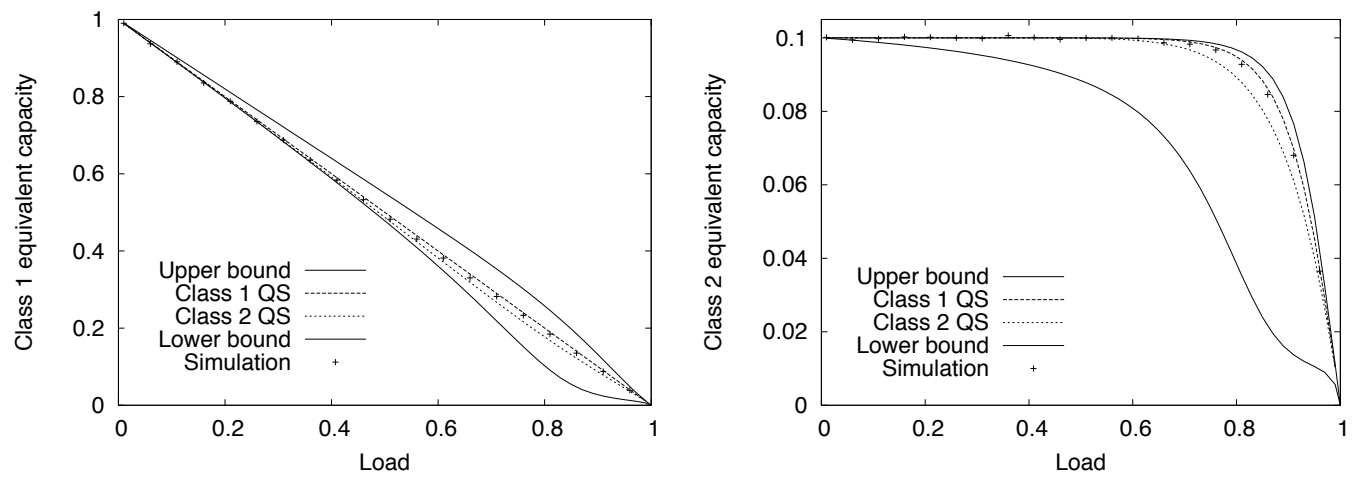

Most load generated by class $2\left(\rho_{1} / \rho=0.1, \rho_{2} / \rho=0.9\right)$
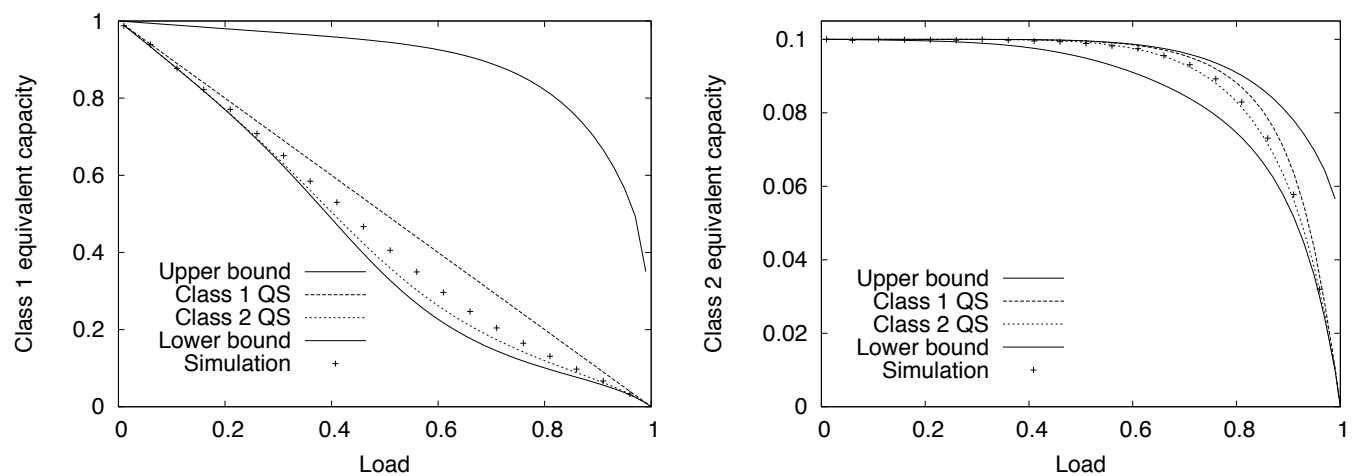

Figure 4: Performance of a 2-class multirate system. $\left(a_{1}=1, a_{2}=0.1\right.$, different load distribution between class 1 and class 2$)$ 
Note that, in view of the limiting regimes, the system looks approximately insensitive except for class 1 when most load is generated by class 2 . If $a_{2}=\frac{1}{n}$ for some integer $n \geq 1$, the equivalent capacities of class 1 in the limiting case $\rho_{1} \sim 0$ when class 1 and class 2 are quasi-stationary are respectively given by:

$$
\bar{\gamma}_{1}^{1}=1-\rho \quad \text { and } \quad \bar{\gamma}_{1}^{2}=\frac{\sum_{k=0}^{n-1} \frac{(n \rho)^{k}}{k !}+\frac{1}{1-\rho} \frac{(n \rho)^{n}}{n !}}{\sum_{k=0}^{n-1} \frac{1}{1-\frac{k}{n}} \frac{(n \rho)^{k}}{k !}+\frac{n(1-\rho)+1}{(1-\rho)^{2}} \frac{(n \rho)^{n}}{n !}} .
$$

It may be verified that the ratio $\bar{\gamma}_{1}^{1} / \bar{\gamma}_{1}^{2}$ takes arbitrarily large values when $n$ increases with $\rho \sim 1-\frac{1}{\sqrt{n}}$. For $n=100$ for instance, we obtain $\bar{\gamma}_{1}^{1}=0.1$ and $\bar{\gamma}_{1}^{2}=\ldots$ for $\rho=0.9$.

\subsection{Concentration tree network with max-min fair sharing}

Finally, we consider a data network that consists of a set of links and a set of $N$ subsets of links referred to as the routes, as considered in $[5,7]$. Let $r_{i}$ be the route of class- $i$ flows. Capacity sharing is max-min fair $[2,20]$. Such a data network can be represented as a PS network where each node corresponds to a particular route. In the simple case of two links 1,2 of respective capacities $C_{1}>C_{2}$ and two routes $r_{1}=\{1\}$ and $r_{2}=\{1,2\}$ for instance, we obtain:

$$
\phi_{1}(x)=1-\phi_{2}(x) \quad \text { with } \quad \phi_{2}(x)=\min \left(C_{2}, \frac{x_{2}}{x_{1}+x_{2}}\right) .
$$

We say that the data network is a concentration tree if the graph formed by the routes has a tree topology and each route consists of a direct path in this graph starting from the root of the tree, as illustrated in Figure 5.

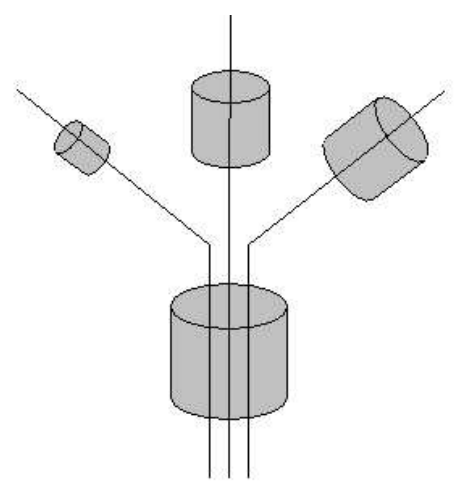

Figure 5: A 3-branch tree

Proposition 5 The PS network is monotonic if and only if the data network is a concentration tree.

Proof. A data network that is not a concentration tree contains either a 2-link line, i.e., a network of two links 1,2 of respective capacities $C_{1} \leq C_{2}$ and three routes $r_{1}=\{1\}, r_{2}=\{2\}$ and $r_{3}=\{1,2\}$, or a triangle, i.e., a network of three links 1,2,3 of respective capacities $C_{1} \leq C_{2} \leq C_{3}$ and three routes 
$r_{1}=\{1,3\}, r_{2}=\{2,3\}$ and $r_{3}=\{1,2\}$. We deduce that the monotonicity property does not hold as in state $x(2,1,2)$, we obtain in both cases,

$$
\phi_{2}(x)=C_{2}-C_{1} / 2 \text { and } \phi_{2}\left(x-e_{1}\right) \leq C_{2}-2 C_{1} / 3
$$

The sufficient condition simply follows from the "filling" procedure of max-min fairness described in $§ 5.3$.

It is worth noting that max-min fairness coincides with proportional fairness [15] and more generally with any utility maximizing allocation in a concentration tree [7]. Note also that the PS network remains monotonic in the presence of additional access rate constraints as considered in $§ 5.3$. Though we expect the stability condition (6) to hold whenever $\rho<1$ for a unit capacity root, we have not been able to prove this result.

The bias property does not hold in general. In fact, it holds only for the particular class of concentration trees known as parking lots. Parking lots are networks with $N$ links of respective capacities $C_{1}>C_{2}>\ldots>C_{N}$ and $N$ routes $r_{1}=\{1\}, r_{2}=\{1,2\}, \ldots, r_{N}=\{1,2, \ldots, N\}$.

Proposition 6 The PS network associated with a parking lot is biased.

Proof. Let $x$ be a state such that $x_{i}>0, x_{j}>0$ for some $i, j$ with $i<j$. If some link $k, i<k \leq j$, is saturated in state $x$, this link is also saturated in state $x-e_{i}$ so that:

$$
\frac{\phi_{i}\left(x-e_{j}\right)}{\phi_{i}(x)} \geq 1=\frac{\phi_{j}\left(x-e_{i}\right)}{\phi_{j}(x)}
$$

Now if no link $k, i<k \leq j$, is saturated in state $x$, we necessarily have

$$
\frac{\phi_{i}(x)}{x_{i}}=\frac{\phi_{j}(x)}{x_{j}}
$$

and the proof follows from the fact that:

$$
\frac{\phi_{i}\left(x-e_{j}\right)}{x_{i}} \geq \frac{\phi_{j}\left(x-e_{i}\right)}{x_{j}}
$$

For a 2-link parking lot, we get:

$$
\check{\Phi}(x)=\frac{1}{C_{2}^{x_{2}}} \prod_{n=1}^{x_{1}} \frac{1}{\max \left(C_{1} \frac{n}{n+x_{2}}, C_{1}-C_{2}\right)} \quad \text { and } \quad \hat{\Phi}(x)=\frac{1}{C_{1}^{x_{1}}} \prod_{n=1}^{x_{2}} \frac{1}{\min \left(C_{1} \frac{n}{x_{1}+n}, C_{2}\right)} .
$$

Figures 6 and 7 show the results obtained for a 2-link parking lot and a 3-branch homogeneous tree, i.e., a tree with one root and three branches of same capacity. Note that, by symmetry, the three limiting regimes coincide in Figure 7. We observe that the lower and upper bounds are very close, indicating that the system is approximately insensitive. This is not always the case, however. As a concentration tree with one single-link route $r_{1}=\{1\}$ and $N-1$ routes $r_{i}=\{1, i\}, i=2, \ldots, N$, all routes having the same traffic intensity $\frac{\rho}{N}$ and all branches the same capacity, tends to a multirate system when $N$ tends to infinity, it follows from $\S 5.3$ that the ratio of the class-1 equivalent capacities of two limiting regimes may take arbitrarily large values at high loads. 

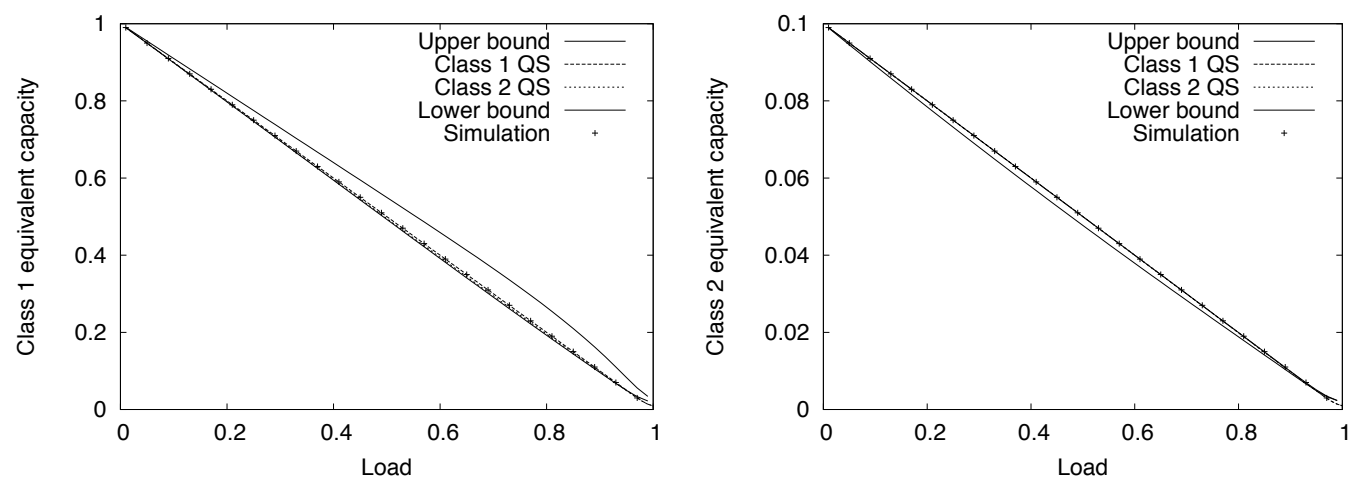

Figure 6: Performance of a 2-link parking lot with max-min fair sharing. $\left(C_{1}=1, C_{2}=0.1, \rho_{1} / \rho=0.9, \rho_{2} / \rho=0.1\right)$

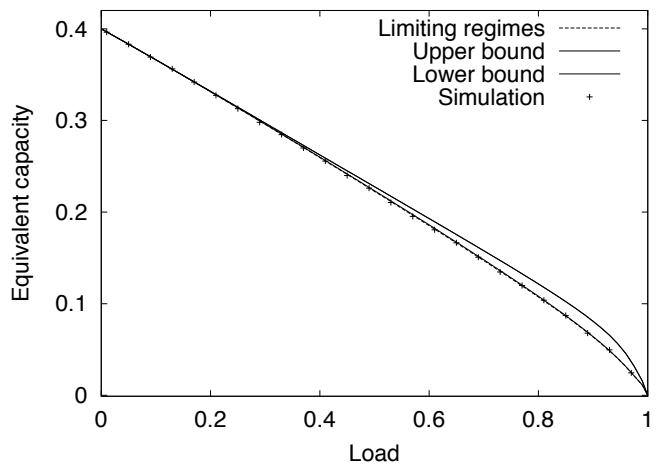

Figure 7: Performance of a 3-branch homogeneous tree with max-min fair sharing. (root capacity $C=1$, branch capacities $C_{1}=C_{2}=C_{3}=0.4, \rho_{1}=\rho_{2}=\rho_{3}$ )

\section{Conclusion}

We have derived stochastic bounds for monotonic PS networks by means of sample-path comparisons with balanced networks. We have also identified the limiting regimes obtained when nodes operate at very different time-scales so as to quantify the sensitivity of the network and to assess the tightness of these bounds. The bounds and the limiting regimes are insensitive to the distribution of service requirements. The lower bound proves tight in cases where most load is generated by "favored" nodes (in the sense of the bias property) while the upper bound proves tight in cases where most load is generated by "penalized" nodes. We have illustrated these results on a number of practically interesting sensitive systems, including the DPS and GPS queues and multirate systems.

A natural question of interest is whether these insensitive bounds can be improved. Numerical results of Section 5 notably suggest that the limiting regimes constitute bounds when the bias property holds. This is only a conjecture, however, which may hold for a weaker order than the considered order $\leq_{s t}$. We are currently working on this subject. 


\section{Appendix}

\section{A Convergence of probability measures}

We say that a family of probability measures $\left\{\pi_{s}, s \geq 0\right\}$ on $\mathbb{N}^{N}$ converges weakly to a probability measure $\pi$ and write $\pi_{s} \Rightarrow \pi$ if:

$$
\forall x \in \mathbb{N}^{N}, \quad \pi(x)=\lim _{s \rightarrow \infty} \pi_{s}(x) .
$$

Denote by $X_{s}$ a random variable with distribution $\pi_{s}, X$ a random variable with distribution $\pi$. For any increasing function $f$, if $E[f(X)]=\infty$, then

$$
\lim _{s \rightarrow \infty} E\left[f\left(X_{s}\right)\right]=\infty .
$$

If $\left\{X_{s}, s \geq 0\right\}$ is $f$-uniformly integrable in the sense that for any $\varepsilon>0$, there exists $n>0$ such that:

$$
\forall s \geq 0, \quad E\left[f\left(X_{s}\right) \mathbb{I}_{\left\{\left|X_{s}\right|>n\right\}}\right]<\varepsilon .
$$

then

$$
\lim _{s \rightarrow \infty} E\left[f\left(X_{s}\right)\right]=E[f(X)]
$$

\section{B Insensitive limiting regimes}

Proof of Theorem 3. We prove that the only possible weak limit $\bar{\pi}$ of $\pi_{\sigma_{1}, \sigma_{2}, \ldots, \sigma_{N}}$ when $\sigma_{1}, \sigma_{2}, \ldots, \sigma_{N-1}$ successively tend to 0 is the probability measure defined by (8). The proof then follows from the tightness of the family of probability measures $\pi_{\sigma_{1}, \ldots, \sigma_{N}}$. We first assume that services are i.i.d. exponential. The stationary distribution $\pi \equiv \pi_{\sigma_{1}, \sigma_{2}, \ldots, \sigma_{N}}$ then satisfies the balance equations:

$$
\sum_{i=1}^{N} \frac{1}{\sigma_{i}}\left(\left(\rho_{i}+\phi_{i}(x)\right) \pi(x)-\rho_{i} \pi\left(x-e_{i}\right)-\phi_{i}\left(x+e_{i}\right) \pi\left(x+e_{i}\right)\right)=0,
$$

where we use the convention $\pi(x)=0$ if $x_{i}<0$ for some $i$. Letting $\sigma_{1}, \sigma_{2}, \ldots, \sigma_{N-1}$ successively tend to 0, we obtain:

$$
\left(\rho_{1}+\phi_{1}(x)\right) \bar{\pi}(x)-\rho_{1} \bar{\pi}\left(x-e_{1}\right)-\phi_{1}\left(x+e_{1}\right) \bar{\pi}\left(x+e_{1}\right)=0 .
$$

Thus for any fixed $x_{2}, \ldots, x_{N}$, if $\bar{\pi}\left(x_{1}, x_{2}, \ldots, x_{N}\right)>0$ for some $x_{1} \geq 0$ then $\bar{\pi}\left(\cdot, x_{2}, \ldots, x_{N}\right)$ is an invariant measure of finite sum for the birth and death process with birth rate $\rho_{1}$ and state dependent death rate $\phi_{1}\left(\cdot, x_{2}, \ldots, x_{N}\right)$. The probability measure $\bar{\pi}_{1}\left(\cdot, x_{2}, \ldots, x_{N}\right)$ is then well defined and:

$$
\bar{\pi}_{1}\left(\cdot, x_{2}, \ldots, x_{N}\right)=\frac{\bar{\pi}\left(\cdot, x_{2}, \ldots, x_{N}\right)}{\sum_{x_{1}} \bar{\pi}\left(x_{1}, x_{2}, \ldots, x_{N}\right)} .
$$

In particular, if $\bar{\pi}\left(x_{1}, x_{2}, \ldots, x_{N}\right)>0$ for some $x_{1} \geq 0$ then $\bar{\pi}\left(x_{1}, x_{2}, \ldots, x_{N}\right)>0$ for all $x_{1} \geq 0$.

Now for any fixed $x_{2}, \ldots, x_{N}$, summing (10) over $x_{1}$ yields:

$$
\sum_{x_{1}} \sum_{i \neq 1} \frac{1}{\sigma_{i}}\left(\left(\rho_{i}+\phi_{i}(x)\right) \pi(x)-\rho_{i} \pi\left(x-e_{i}\right)-\phi_{i}\left(x+e_{i}\right) \pi\left(x+e_{i}\right)\right)=0,
$$

where we used the fact that:

$$
\sum_{x_{1}}\left(\rho_{1}+\phi_{1}(x)\right) \pi(x)=\sum_{x_{1}}\left(\pi\left(x-e_{i}\right) \rho_{1}+\phi_{i}\left(x+e_{i}\right) \pi\left(x+e_{i}\right)\right) .
$$


Letting $\sigma_{1}, \sigma_{2}, \ldots, \sigma_{N-1}$ successively tend to 0 , we obtain:

$$
\sum_{x_{1}}\left(\left(\rho_{2}+\phi_{2}(x)\right) \bar{\pi}(x)-\rho_{2} \bar{\pi}\left(x-e_{2}\right)-\phi_{2}\left(x+e_{2}\right) \bar{\pi}\left(x+e_{2}\right)\right)=0 .
$$

Thus for any fixed $x_{3}, \ldots, x_{N}$, if $\sum_{x_{1}} \bar{\pi}\left(x_{1}, x_{2}, \ldots, x_{N}\right)>0$ for some $x_{2} \geq 0$ then $\sum_{x_{1}} \bar{\pi}\left(x_{1}, \cdot, x_{3}, \ldots, x_{N}\right)$ is an invariant measure for the birth and death process with birth rate $\rho_{2}$ and state dependent death rate $\bar{\phi}_{2}\left(\cdot, x_{3}, \ldots, x_{N}\right)$. We then deduce that the probability measure $\bar{\pi}_{2}\left(\cdot, x_{3}, \ldots, x_{N}\right)$ is well defined and:

$$
\bar{\pi}_{2}\left(\cdot, x_{3}, \ldots, x_{N}\right)=\frac{\sum_{x_{1}} \bar{\pi}\left(x_{1}, \cdot, x_{3}, \ldots, x_{N}\right)}{\sum_{x_{1}, x_{2}} \bar{\pi}\left(x_{1}, x_{2}, \ldots, x_{N}\right)} .
$$

In particular, if $\bar{\pi}\left(x_{1}, x_{2}, \ldots, x_{N}\right)>0$ for some $x_{1}, x_{2} \geq 0$ then $\bar{\pi}\left(x_{1}, x_{2}, \ldots, x_{N}\right)>0$ for all $x_{1}, x_{2} \geq 0$.

Applying successively the same reasoning for $i=3, \ldots, N-1$, we deduce that for any fixed $x_{i+1}, \ldots, x_{N}$, if $\sum_{x_{1}, \ldots, x_{i-1}} \bar{\pi}\left(x_{1}, \ldots, x_{i}, \ldots, x_{N}\right)>0$ for some $x_{i} \geq 0$, then $\sum_{x_{1} \ldots, x_{i-1}} \bar{\pi}\left(x_{1}, \ldots, x_{i-1}, \cdot, x_{i+1}, \ldots, x_{N}\right)$ is an invariant measure for the birth and death process with birth rate $\rho_{i}$ and state dependent death rate $\bar{\phi}_{i}\left(\cdot, x_{i+1}, \ldots, x_{N}\right)$. We then deduce that the probability measure $\bar{\pi}_{i}\left(\cdot, x_{i+1}, \ldots, x_{N}\right)$ is well defined and:

$$
\bar{\pi}_{i}\left(\cdot, x_{i+1}, \ldots, x_{N}\right)=\frac{\sum_{x_{1}, \ldots, x_{i-1}} \bar{\pi}\left(x_{1}, \ldots, x_{i-1}, \cdot, x_{i+1}, \ldots, x_{N}\right)}{\sum_{x_{1}, \ldots, x_{i}} \bar{\pi}\left(x_{1}, \ldots, x_{i-1}, x_{i}, x_{i+1}, \ldots, x_{N}\right) .}
$$

In particular, if $\bar{\pi}\left(x_{1}, \ldots, x_{i}, \ldots, x_{N}\right)>0$ for some $x_{1}, \ldots, x_{i} \geq 0$ then $\bar{\pi}\left(x_{1}, \ldots, x_{i}, \ldots, x_{N}\right)>0$ for all $x_{1}, \ldots, x_{i} \geq 0$.

Now for each fixed $x_{N}$, summing (10) over $x_{1}, \ldots, x_{N-1}$ yields:

$$
\sum_{x_{1}, \ldots, x_{N-1}}\left(\left(\rho_{N}+\phi_{N}(x)\right) \pi(x)-\rho_{N} \pi\left(x-e_{N}\right)-\phi_{N}\left(x+e_{N}\right) \pi\left(x+e_{N}\right)\right)=0 .
$$

Letting $\sigma_{1}, \sigma_{2}, \ldots, \sigma_{N-1}$ successively tend to 0 , we deduce that these balance equations are also satisfied by $\bar{\pi}$. Thus if $\sum_{x_{1}, \ldots, x_{N-1}} \bar{\pi}\left(x_{1}, \ldots, x_{N-1}, x_{N}\right)>0$ for some $x_{N} \geq 0, \sum_{x_{1}, \ldots, x_{N-1}} \bar{\pi}\left(x_{1}, \ldots, x_{N-1}, \cdot\right)$ is an invariant measure for the birth and death process with birth rate $\rho_{N}$ and state dependent death rate $\bar{\phi}_{N}$ and:

$$
\bar{\pi}_{N}(\cdot)=\frac{\sum_{x_{1}, \ldots, x_{N-1}} \bar{\pi}\left(x_{1}, \ldots, x_{N-1}, \cdot\right)}{\sum_{x_{1}, \ldots, x_{N}} \bar{\pi}\left(x_{1}, \ldots, x_{N}\right)} \equiv \sum_{x_{1}, \ldots, x_{N-1}} \bar{\pi}\left(x_{1}, \ldots, x_{N-1}, \cdot\right) .
$$

In particular, if $\bar{\pi}\left(x_{1}, x_{2}, \ldots, x_{N}\right)>0$ for some $x_{1}, \ldots, x_{N} \geq 0$ then $\bar{\pi}\left(x_{1}, x_{2}, \ldots, x_{N}\right)>0$ for all $x_{1}, \ldots, x_{N} \geq 0$. As $\bar{\pi}$ is a probability measure, $\bar{\pi}(x)>0$ for some $x \geq 0$ so that $\bar{\pi}(x)>0$ for all $x \geq 0$. Expression (8) follows from the above relations between $\bar{\pi}$ and $\bar{\pi}_{i}$, for $i=1, \ldots, N$.

Now assume that the service requirements at each node are i.i.d. with a Cox distribution. We shall demonstrate that the limiting distribution of the number of customers at each node is still given by (8). The proof then follows from the fact that Cox distributions form a dense subset within the set of all distributions of nonnegative random variables. Services at node $i$ consist of $M_{i}$ exponential phases of respective means $\sigma_{i} / \mu_{i j}, j=1, \ldots, M_{i}$. At the end of the $j$-th phase, a service at node $i$ enters the $(j+1)$-th phase with probability $p_{i j}$ and ends with probability $1-p_{i j}$ (with $p_{i M_{i}} \equiv 0$ ). We denote by $y_{i j}$ the number of customers at node $i$ whose service is in phase $j$. The stochastic process $\{Y(t)\}_{t \geq 0}$ describing the evolution of the network state $y=\left(y_{i j}, i=1, \ldots, N ; j=1, \ldots, M_{i}\right)$ is an irreducible 
Markov process for which the balance equations write:

$$
\begin{aligned}
& \sum_{i=1}^{N} \frac{1}{\sigma_{i}}\left\{\left(\rho_{i}+\sum_{j=1}^{M_{i}} \psi_{i j}(y) \mu_{i j}\right) \pi(y)-\rho_{i} \pi\left(y-f_{i 1}\right)\right. \\
& \left.\quad-\sum_{j=1}^{M_{i}} \psi_{i j}\left(y-f_{i j+1}+f_{i j}\right) \mu_{i j} p_{i j} \pi\left(y-f_{i j+1}+f_{i j}\right)-\sum_{j=1}^{M_{i}} \psi_{i j}\left(y+f_{i j}\right) \mu_{i j}\left(1-p_{i j}\right) \pi\left(y+f_{i j}\right)\right\}=0,
\end{aligned}
$$

where $f_{i j}$ is the unit vector corresponding to a single customer at node $i$ with a service in phase $j$, and $\psi_{i j}$ is the service rate of the $y_{i j}$ customers at node $i$ with a service in phase $j$ :

$$
\psi_{i j}(y)=\frac{y_{i j}}{x_{i}} \phi_{i}(x), \quad \text { with } \quad x_{i}=\sum_{j=1}^{M_{i}} y_{i j} .
$$

Applying the same reasoning as above, it follows from the insensitivity of the successively considered birth and death processes that any weak limit $\bar{\pi}$ of the probability measure $\pi$, obtained by letting $\sigma_{1}, \ldots, \sigma_{N-1}$ successively tend to 0 , satisfies:

$$
\sum_{y: \forall i, \sum_{j=1}^{M_{i}} y_{i j}=x_{i}} \bar{\pi}(y)=\prod_{i=1}^{N} \bar{\pi}_{i}\left(x_{i}, \ldots, x_{N}\right) .
$$

Proof of Theorem 4. We assume without loss of generality that $\bar{\pi}$ is the limiting distribution obtained when $\sigma_{1}, \sigma_{2}, \ldots, \sigma_{N-1}$ successively tend to 0 , with fixed traffic intensities $\rho_{1}, \rho_{2}, \ldots, \rho_{N-1}$. Let $\bar{\pi}_{i}$ and $\bar{\phi}_{i}$ be defined as in Theorem 3 , for $i=1, \ldots, N$. Note that $\bar{\pi}_{i}$ depends on the traffic intensities $\rho_{1}, \ldots, \rho_{i}$ only. We prove that the probability measure $\bar{\pi}_{i}\left(\cdot, x_{i+1}, \ldots, x_{N}\right)$ increases with respect to $\rho_{1}, \ldots, \rho_{i}$ and $x_{i+1}, \ldots, x_{N}$, in the sense of the stochastic order $\leq_{s t}$. The proof is by induction on $i$. The property holds for $i=1$ in view of the monotonicity property. Let $i \geq 2$ and assume the property holds for $j=1, \ldots, i-1$. Writing

$$
\bar{\phi}_{i}\left(x_{i}, \ldots, x_{N}\right)=\sum_{x_{i-1}} \bar{\pi}_{i-1}\left(x_{i-1}, \ldots, x_{N}\right) \sum_{x_{i-2}} \bar{\pi}_{i-2}\left(x_{i-2}, \ldots, x_{N}\right) \ldots \sum_{x_{1}} \bar{\pi}_{1}\left(x_{1}, \ldots, x_{N}\right) \phi_{i}(x),
$$

and applying successively the property for $j=1, \ldots, i-1$, we deduce from the monotonicity property that the function $\bar{\phi}_{i}\left(\cdot, x_{i+1}, \ldots, x_{N}\right)$ decreases with respect to $\rho_{1}, \ldots, \rho_{i-1}$ and $x_{i+1}, \ldots, x_{N}$. The property then holds for $i$ since $\bar{\pi}_{i}\left(\cdot, x_{i+1}, \ldots, x_{N}\right)$ is the stationary distribution of a birth and death process with birth rate $\rho_{i}$ and death rate $\bar{\phi}_{i}\left(\cdot, x_{i+1}, \ldots, x_{N}\right)$. Now for any increasing function $f$, writing

$$
E[f(\bar{X})]=\sum_{x_{N}} \bar{\pi}_{N}\left(x_{N}\right) \sum_{x_{N-1}} \bar{\pi}_{N-1}\left(x_{N-1}, x_{N}\right) \ldots \sum_{x_{1}} \bar{\pi}_{1}\left(x_{1}, \ldots, x_{N}\right) f(x),
$$

and applying successively the above property for $i=1, \ldots, N$, we deduce that $E[f(\bar{X})]$ is an increasing function of $\rho_{1}, \ldots, \rho_{N}$. In particular, $E[f(\bar{X})]$ decreases to $E\left[f\left(Y_{N} e_{N}\right)\right]$ when $\rho_{1}, \ldots, \rho_{N-1}$ tend to 0 .

If $E[f(\check{X})]<\infty$, we have

$$
E\left[f(\check{X}) \mathbb{I}_{\{|\check{X}|>n\}}\right]=\frac{\sum_{x:|x|>n} f(x) \check{\Phi}(x) \prod_{i=1}^{N} \rho_{i}^{x_{i}}}{\sum_{x} \check{\Phi}(x) \prod_{i=1}^{N} \rho_{i}^{x_{i}}} .
$$


Thus for any $\varepsilon>0$, there exists $n \geq 0$ such that for any traffic intensities smaller than $\rho_{1}, \ldots, \rho_{N}$,

$$
E\left[f(\check{X}) \mathbb{I}_{\{|\check{X}|>n\}}\right]<\varepsilon .
$$

In view of Theorem 1, Corollary 1 and Proposition 3, we obtain

$$
E\left[f(\bar{X}) \mathbb{I}_{\{|\bar{X}|>n\}}\right] \leq E\left[f(\check{X}) \mathbb{I}_{\{|\check{X}|>n\}}\right]<\varepsilon .
$$

Now if for some $j \neq N, f(x)=0$ for all states $x$ such that $x_{j}=0$, we have

$$
\frac{E\left[f(\bar{X}) \mathbb{I}_{\{|\bar{X}| \leq n\}}\right]}{\rho_{j}}=\sum_{x:|x| \leq n, x_{j}>0} \bar{\pi}_{N}\left(x_{N}\right) \ldots \bar{\pi}_{j}\left(0, x_{j+1}, \ldots, x_{N}\right) \frac{\rho_{j}^{x_{j}-1}}{\prod_{y=1}^{x_{j}} \bar{\phi}_{j}\left(y, x_{j+1}, \ldots, x_{N}\right)} \ldots \bar{\pi}_{1}\left(x_{1}, \ldots, x_{N}\right) f(x) .
$$

We know that for any $i<N$, the probability measure $\bar{\pi}_{i}\left(\cdot, x_{i+1}, \ldots, x_{N}\right)$ decreases to the Dirac measure in 0 when $\rho_{1}, \ldots, \rho_{i}$ decrease to 0 while the function $\bar{\phi}_{j}\left(\cdot, x_{i+1}, \ldots, x_{N}\right)$ tends to $\phi_{j}\left(0, \ldots, 0, \cdot, x_{i+1}, \ldots, x_{N}\right)$ when $\rho_{1}, \ldots, \rho_{j-1}$ tend to zero. As the probability measure $\bar{\pi}_{N}(\cdot)$ decreases to $\zeta_{N}$ when $\rho_{1}, \ldots, \rho_{N-1}$ decrease to 0 , we obtain for sufficiently small traffic intensities $\rho_{1}, \ldots, \rho_{N-1}$,

$$
\left|\frac{E\left[f(\bar{X}) \mathbb{I}_{\{|\bar{X}| \leq n\}}\right]}{\rho_{j}}-\sum_{y=0}^{n-1} \frac{f\left(y e_{N}+e_{j}\right)}{\phi_{j}\left(y e_{N}+e_{j}\right)} \zeta_{N}(y)\right|<\varepsilon
$$

We conclude that

$$
\lim _{\rho_{i} \rightarrow 0, i \neq N} \frac{E[f(\bar{X})]}{\rho_{j}}=\sum_{y=0}^{\infty} \frac{f\left(y e_{N}+e_{j}\right)}{\phi_{j}\left(y e_{N}+e_{j}\right)} \zeta_{N}(y)
$$

\section{References}

[1] F. Baskett, K.M. Chandy, R.R. Muntz and F.G. Palacios, Open, closed and mixed networks of queues with different classes of customers, J. Assoc. Comput. Mach. 22 (1975) 248-260.

[2] D. Bertsekas and R. Gallager, Data Networks, Prentice Hall, 1987.

[3] D. Bertsimas, I. C. Paschalidis, J. N. Tsitsiklis, Large deviation analysis of the Generalized Processor Sharing policy, Queueing Systems 32 (1999) 319-349.

[4] P. Billingsley, Convergence of Probability Measures, Wiley, New York, 1968.

[5] T. Bonald and L. Massoulié, Impact of fairness on Internet performance, in: Proc. of ACM SIGMETRICS, 2001.

[6] T. Bonald and A. Proutière, Insensitivity in processor-sharing networks, Performance Evaluation 49 (2002) 193-209.

[7] T. Bonald and A. Proutière, Insensitive bandwidth sharing in data networks, Queueing Systems 44 (2003) 69-100 .

[8] S. Borst, O.J. Boxma, P. Jelenkovic, Reduced-load equivalence and induced burstiness in GPS queues with long-tailed traffic flows, Queueing Systems 43-4 (2003) 273-306. 
[9] G. L. Choudhury, A. Mandelbaum, M. I. Reiman, W. Whitt, Fluid and Diffusion Limits for Queues in Slowly Changing Environments, Stochastic Models 13 (1997) 121-146.

[10] J.W. Cohen, The multiple phase service network with generalized processor sharing, Acta Informatica 12 (1979) 245-284.

[11] A. Dembo, O. Zeitouni, Large Deviations Techniques and Applications, Jones and Barlett, 1993.

[12] G. Fayolle, I. Mitrani and R. Iasnogorodski, Sharing a processor among many classes, Journal of the ACM 27 (1980) 519-532.

[13] P.R. Jelenkovic, The effect of multiple time scales and subexponentiality on the behavior of a broadband network multiplexer, $\mathrm{PhD}$ thesis, Columbia University, New York, October 1996.

[14] F.P. Kelly, Reversibility and Stochastic Networks, Wiley, 1979.

[15] F.P. Kelly, A. Maulloo and D. Tan, Rate control for communication networks: Shadow prices, proportional fairness and stability, Journal of the Operational Research Society 49 (1998) 237-252.

[16] F.P. Kelly and R.J. Williams, Fluid models for a network operating under a fair bandwidth-sharing policy, submitted for publication, available at:

http://www.math.ucsd.edu/ williams/bandwidth/kwfluid.html

[17] L. Kleinrock, Queueing Systems, Volume 2, Wiley, 1975.

[18] H.J. Kushner, Heavy-Traffic of controlled Queueing and Commmunication Networks, Springer, 2001.

[19] L. Massoulié, Large Deviation estimates for polling and Weighted Fair Queueing systems, Advances in Performance Analysis (1999).

[20] L. Massoulié and J.W. Roberts, Bandwidth sharing: objectives and algorithms, IEEE/ACM Transactions on Networking 10-3 (2002) 320-328.

[21] L. Massoulié and J.W. Roberts, Bandwidth sharing and admission control for elastic traffic, Telecommunication Systems 15 (2000) 185-201.

[22] R. Núñez Queija, Processor-Sharing Models for Integrated-Services Networks, PhD Thesis, Eindhoven University of Technology, 1999.

[23] R.F. Serfozo, Introduction to Stochastic Networks, Springer, 1999.

[24] G.G. Yin, Q. Zhang, Continuous-Time Markov Chains and Applications: A singular perturbation approach, Springer, 1998.

[25] W. Whitt, Stochastic-Process Limits: An introduction to stochastic-process limits and their applications to queues, Springer, 2002. 